\title{
nature
}

\section{Nature's 2001}

This publication is set to be ever more proactive in its development of content and services. But reflections are appropriate as we finally enter a new millennium, as well as some statements of commitment.

$N$ ature's primary aim every week, since our beginnings in 1869 , has been and will be to keep researchers and society at large in touch with the state of the best of scientific research and the people who do it. One important secondary aim, to keep finding ways to do that better, will also continue unabated.

In achieving those goals, Nature's independence of any scientific organization remains one of our most important assets. We believe it maximizes our ability to respond rapidly to new developments and opportunities, and also that it guarantees a precious freedom to publish heterodox views - our own or others' — in an unrestrained but responsible manner.

Another asset, and a corollary of our independence, is our internationalism. Although Nature's content strongly reflects the United States' great and increasing strength in the world of science, no one could complain at the high proportion of our authors from, and of pages devoted to, other countries. Our continuing commitment to that balancing act is reflected by our organizing of a conference last month on Central and Eastern Europe while also deciding to open, this month, an editorial office in San Francisco. In addition, we are playing a leading role in a collaborative project developing free online facilities for scientists in the developing world. And our circulation, which has grown continuously by about $20 \%$ to 64,000 over the past four years and shows no sign of reaching a plateau, reflects this internationalism and regional strength, with $50 \%$ in the United States.

Nature has recently grown significantly more proactive in its commissioning of outstanding authors. Launched in the middle of last year, the series of monthly 'Insight' collections of review articles has boosted enormously the amount of authoritative overview we deliver to readers, while the number of traditional review articles has also grown. And in a more forward-looking spirit, later this month we will publish an Insight survey of future science and technologies, complementing our supplement Impacts of Foreseeable Science published in December 1999.

\section{Innovative writing}

We will also build on another of last year's innovations: the launch of weekly News Features, greatly expanding our coverage of the research community as it responds to key scientific developments. This aspect of editorial proactivity is set to increase as more investment in topquality journalism enhances our provision yet further over the next few months.

Over the past year or two, essays in Nature have provided a welcome complement to the traditional fare of comment, papers and news. To judge by readers' responses, the series of Millennium Essays has well fulfilled our original hopes of providing stimulating accounts of littleknown gems of history. The Futures series - essays too, albeit fictional - has entertained in its own unorthodox way, right up to the final example at the end of last year. The Millennium series will end later this month, and will be immediately followed by the launch of two new series. (Essay series in Nature were initiated by Martin Kemp's on art, image and science, now collected and embellished in a book entitled Visualisations: The Nature Book of Art and Science, published by Oxford
University Press and University of California Press, Berkeley.)

Our essays have also had the merit of being widely readable something not to be taken for granted in a scientific periodical. Indeed, readability is something Nature has cultivated systematically over the past few years. Although there are limits to what can be achieved in a concise space with interdisciplinary communication, we are committed to enhancing yet further the readability of our more technical sections, including our original papers. (Readers may not recognize the enhancements in readability that we inject into our papers' introductions, but believe us, things could be much, much worse.) And readability is not only about words. The comprehensibility and impact of much of our content have been greatly enhanced by imaginative design and improved illustrations.

\section{Service cultures}

Other areas of investment have been in editorial services to authors. Following the introduction of electronic submission last year, the proportion of authors using that route grew rapidly and dramatically to a considerable majority. We have extended electronic refereeing and communication to all manuscripts, whether submitted electronically or not. That, combined with the developing ability to publish selected papers promptly on the web, is speeding up our publication times significantly. Some papers of particularly great public interest have been made freely available on our website ahead of print publication. Online readers will also have noticed improvements to our website within the past month - of which there are more to come.

The quality of research that we publish continues to be paramount, and the resources devoted to the selection process continue to grow. In that regard, although impact factors are by no means everything, we do enjoy the fact that ours is the highest among the general science journals.

Competitors are always with us and help keep us on our mettle. The newest — but still unresolved — agents of potential change are those who make published papers freely available on the web. Anyone who has examined those activities will realize that at least some of those publishers have not yet achieved a sustainable financial model. For our part, Nature and our publishers stand by a policy of reasonable pricing for high quality content and services. We stand by the value we add all through the publishing process, and the professionalism and responsiveness that a well resourced and dedicated editorial team can bring. However, we are examining ideas of free access as much as an opportunity as they are a challenge.

Questions about access to original research are part of a wider debate about the ethics, politics and economics of public and private ownership in science in all its aspects. This contentious set of issues will be affected by decisions in society as well as the science community: decisions about protection of intellectual property and of data, but also about a public and political resolve in further increasing the public funding of research, amid burgeoning scientific opportunities and related societal controversies. One way or another, publishers are not the only participants in these debates facing challenges to their assumptions in the near future. 